\title{
ESTIMATIVA DA DEPOSIÇÃO DE AGROTÓXICOS POR ANÁLISE DE GOTAS ${ }^{1}$
}

\author{
ALDEMIR CHAIM ${ }^{2}$, ALINE HOLANDA NUNES MAIA ${ }^{3}$ e MARIA CONCEIÇÃO PERES YOUNG PESSOA ${ }^{4}$
}

\begin{abstract}
RESUMO - As quantificações dos agrotóxicos que atingem os alvos são feitas com metodologias onerosas ou sofisticadas. Assim, o objetivo deste trabalho foi comparar dois métodos para quantificar as deposições de agrotóxicos: um, por análise de gotas, e o outro, por análise química de traçador. As gotas de uma calda cúprica foram amostradas com papel sensível a água, lâmina de microscópio recoberta com óxido de magnésio e cartões de papel-filtro. No método de análise de gotas, o volume depositado em determinada área foi calculado considerando-se os diâmetros das manchas ou impressões, fatores de espalhamento e densidade de deposição. No outro método, o volume depositado foi obtido mediante análise de resíduo do traçador encontrado nos alvos e pela concentração da calda da pulverização. Não houve diferenças estatísticas entre os volumes estimados pelos dois métodos.
\end{abstract}

Termos para indexação: pulverização, gotas, deposição, método de recuperação de deposição.

\section{ESTIMATES OF PESTICIDE DEPOSITION BY DROPLET SIZE ANALYSIS}

\begin{abstract}
The available methodologies for analysing the pesticide reaching the target are sophisticated or expensive. The objective of this work was to compare two methods to quantify the pesticide deposition. One of them by droplets analysis and the other by chemical tracer analysis. The droplets of a fungicide spray were sampled using water sensitive papers, slides with oxide magnesium and cards of filter paper. In the droplet analysis method, the volume of spray deposited on a delimited area was estimated considering the diameters of stains or craters, spread factors and droplet density. In the chemical tracer analysis method the volume was calculated by the residue deposited on sampling target and spray concentration. There were no statistic differences in the volumes estimated by the two methods.
\end{abstract}

Index terms: spraying, droplets, recovery, method for deposition recovery.

\section{INTRODUÇÃO}

Os métodos empregados para quantificação da deposição das aplicações têm utilizado análises químicas de traçantes, ou dos próprios agrotóxicos utilizados nos tratamentos fitossanitários. Na maioria das vezes, esses métodos são caros e pouco acessíveis às empresas de aplicação ou mesmo às instituições de pesquisas que atuam na área de tecnologia de aplicação.

\footnotetext{
${ }^{1}$ Aceito para publicação em 16 de setembro de 1998.

${ }^{2}$ Eng. Agr., M.Sc., Embrapa-Centro Nacional de Pesquisa de Monitoramento e Avaliação de Impacto Ambiental (CNPMA), Caixa Postal 69, CEP 13820-000 Jaguariúna, SP. E-mail: aldemir@cnpma.embrapa.br

${ }^{3}$ Eng $\underline{a}$ Agr ${ }^{\mathrm{a}}$, M.Sc., Embrapa-CNPMA.

${ }^{4}$ Matemática Aplicada, Ph.D., Embrapa-CNPMA.
}

Alguns pesquisadores têm utilizado sistema computadorizado para medir gotas. Normalmente, esse sistema utiliza-se de imagens de gotas, amostradas em cartões ou folhas de plantas, capturadas por uma câmera de vídeo ou scanners de computadores. As imagens são digitalizadas e os padrões dos depósitos são analisados por softwares específicos. Franz (1993) desenvolveu um sistema que utiliza scanner com resolução de $63,5 \mu \mathrm{m} /$ pixel e obteve medições precisas de manchas de gotas amostradas em papel Kromekote, dentro de uma faixa de tamanho entre 210 a $1.050 \mu \mathrm{m}$. Derksen $\&$ Jiang (1995) desenvolveram um sistema automático, computadorizado, para caracterização de depósitos de gotas de traçantes fluorescentes, em alvos artificiais ou folhas de plantas. Esse sistema foi fundamentado em um processamento morfológico de ima- 
gens de depósitos de gotas, descrito por Jiang \& Derksen (1995). As imagens foram capturadas por uma câmera CCD monocromática, e o software utilizado permitiu medir manchas de gotas entre 5 e $1.000 \mu \mathrm{m}$.

A escolha de um método mais simples e mais barato deve considerar a disponibilidade de equipamentos comuns para análise, como, por exemplo, um microscópio para medir gotas de pulverização. $\mathrm{O}$ volume de cada gota pode ser expresso por $\pi \mathrm{D}^{3 / 6}$, onde D é o diâmetro da gota e pode ser medido com microscópio. Conhecendo-se o diâmetro e o número de gotas que se depositam sobre uma área conhecida, é possível estimar o volume de líquido total presente nessa superfície. Por outro lado, conhecendo-se a concentração da calda de agrotóxico aplicada em algum tipo de alvo específico é também possível estimar o nível de resíduo presente na amostra.

As gotas podem ser amostradas em vários tipos de superfícies artificiais, para, posteriormente, serem medidas com microscópio. Uma superfície considerada como padrão para amostragem de gotas, é obtida pela queima de tiras de magnésio metálico sob superfície de um vidro plano (Matthews, 1982). Gotas com diâmetro entre 20 e 200 Mm têm apresentado uma relação constante entre o diâmetro da cratera formada no óxido de magnésio e o diâmetro original da gota. No óxido de magnésio, as crateras formadas por gotas de água ou outros líquidos são, em média, 1,15 vez maiores que o diâmetro original das gotas (May, 1950). Gotas entre 15-20 e 10-15 $\mu \mathrm{m}$ apresentam fator de conversão na ordem de 0,8 e 0,75 respectivamente. Entretanto, Maksymiuk \& Moore (1962) encontraram que o fator de espalhamento de 1,15 (ou fator de conversão de 0,86 ) proporciona uma correlação linear entre gotas esféricas e manchas de gotas entre 40 a $1.000 \mu \mathrm{m}$. O método do óxido de magnésio não é adequado para gotas menores do que $10 \mu \mathrm{m}$, devido ao pequeno peso que essas partículas apresentam para sedimentação. Por outro lado, gotas maiores que $300 \mu \mathrm{m}$ podem danificar a delicada camada do material, ou se arrebentarem com o impacto, originando gotas menores que podem mascarar o resultado da análise.

Em experimentos de campo, têm sido usadas superfícies alternativas para amostragens de gotas, uma vez que o óxido de magnésio pode ser facilmente danificado. Superfícies como o papel Kromekote
(Higgins, 1967) ou papel fotográfico, têm se demonstrado mais adequadas para a amostragem das gotas. Nesses casos, alguns corantes como a croceína vermelha ou a negrosina têm sido adicionados às caldas para visualização das manchas. Para evitar a contaminação de áreas não-alvos com corantes, foram desenvolvidos métodos para impregnar papéis com corantes, especiais que revelam as gotas que atingem sua superfície. Turner \& Huntington (1970) descrevem um método simples para obtenção de papéis sensíveis a água. Os papéis são impregnados com o corante azul de bromofenol, que na sua forma não-ionizada apresenta coloração amarela. A água, entretanto, ioniza a substância, e esta adquire uma forte coloração azul. Esses papéis estão disponíveis no mercado.

Não existem muitas informações na literatura sobre o emprego da análise de gotas para os estudos de recuperação de agrotóxicos em alvos artificiais. Um dos únicos trabalhos nessa linha, desenvolvido por Corrêa et al. (1992), apresenta uma grande discrepância entre os resultados da análise de gotas e análise química. Os autores confirmam que o método analítico é mais preciso, mas reconhecem que existe uma incoerência entre os resultados.

Assim, o objetivo do trabalho foi testar um método de recuperação de deposição por análise de gotas, utilizando dois alvos distintos, papel sensível a água, e lâmina de vidro recoberta com óxido de magnésio.

\section{MATERIAL E MÉTODOS}

O experimento consistiu na pulverização concomitante de cartões de papel sensível a água, lâminas de microscópio recobertas com $\mathrm{MgO}$, e cartões de papel-filtro, com $0,5 \mathrm{~mm}$ de espessura $\left(250 \mathrm{~g} / \mathrm{m}^{2}\right)$, com uma calda contendo $1.000 \mathrm{mg} / \mathrm{L}$ de cobre metálico. Todas as superfícies apresentavam as dimensões semelhantes às da lâmina de microscópio. Sobre uma mesa foi montada uma fileira contendo 10 superfícies de amostragem de cada tipo, dispostas em triplicata, de maneira que ficassem uma ao lado da outra e posicionadas com o maior comprimento paralelo à direção da fileira.

A pulverização foi feita com um micropulverizador pneumático manual, desenvolvido na Embrapa-Centro Nacional de Pesquisa de Monitoramento e Avaliação de Impacto Ambiental (CNPMA), com jato de padrão cone vazio (ângulo aproximado de $80^{\circ}$ ), com vazão constante de $6,1 \mathrm{~mL} / \mathrm{min}$, em pressão de ar calibrada para $13,78 \mathrm{kPa}$. 
de superfície de amostragem, até a obtenção de um coeficiente de variação de apenas $2 \%$, entre os números de gotas das classes, em 4 classificações sucessivas. Após esse treinamento foram feitas as classificações das gotas amostradas, nos 20 alvos empregados no teste. $\mathrm{O}$ mesmo procedimento de treinamento foi adotado para determinação da densidade de gotas, com a diferença de que foram delimitados dois campos de $1 \mathrm{~cm}^{2}$ em cada superfície de amostragem, onde as sucessivas contagens foram realizadas. Após o treinamento, procedeu-se a determinação da densidade de gotas nas amostras, em áreas demarcadas. Foram feitas contagens, em três campos diferentes de cada amostra, e a densidade de gotas foi obtida pela média entre os números encontrados.

Os valores dos fatores de espalhamento para cada tamanho de impressão no papel sensível a água foram extraídos de um gráfico apresentado num catálogo, sobre uso de papel sensível, distribuído pela Ciba-Geigy (19--). O gráfico original foi ampliado para uma melhor visualização dos pontos de interseção, e foram determinados os valores dos fatores de espalhamento para manchas com diâmetros de: $30,50,100,150,200,250,300,350,400,450,500$, $550,600,650,700,750,800,850,900,950,1.000,1.050$, $1.100 \mu \mathrm{m}$. Os valores intermediários entre 30 e 50, 100 e $150 \mu \mathrm{m}$ e os subseqüentes, foram calculados de $10 \mathrm{em} 10$ micrômetros, pela progressão aritmética entre dois pontos consecutivos. Com esses dados e com a utilização do programa SAS, estabeleceu-se a equação da curva que melhor se ajustasse aos valores.

Os valores dos diâmetros-limite de cada classe de gotas foram divididos pelos fatores de espalhamento correspondentes, após o que, determinou-se o diâmetro médio das classes de tamanho, calculando-se a média entre os limites superior e inferior de cada classe.

Calculou-se o volume de cada gota de diâmetro médio, aplicando-se a fórmula: $\mathrm{V}_{\mathrm{dm}}=\pi \cdot \mathrm{dm}^{3} / 6\left(\mu \mathrm{m}^{3}\right)$. Para obter os valores diretamente em microlitros, dividiu-se o resultado por 1.000.000.000: $\mathrm{V}_{\mathrm{dm}}=\pi . \mathrm{dm}^{3} / 6.000 .000 .000$.

Multiplicou-se o número de gotas encontrada em cada classe, pelo seu volume médio, obtendo-se os volumes parciais para cada classe de tamanho de gotas da amostra analisada. Esses volumes parciais foram somados, obtendo-se o volume total das gotas, o qual foi dividido pelo número total de gotas medidas, obtendo-se, assim, o volume médio das gotas da amostra. Multiplicando-se o volume médio pela densidade de gotas, obteve-se o volume médio por $\mathrm{cm}^{2}$.

Para as lâminas recobertas com $\mathrm{MgO}$, utilizou-se o fator de espalhamento de 1,15 para todas as classes de tamanho, porque, conforme Maksymiuk \& Moore (1962), esse fator é constante para gotas entre 40 e $1.000 \mu \mathrm{m}$.
A Tabela 1, apresenta, como exemplo, os cálculos efetuados para estimar os volumes de calda que atingiram os alvos. São apresentados os resultados para as distribuições das gotas encontradas nas amostras $n^{\underline{0}} 1$ do papel sensível a água e lâmina de microscópio com $\mathrm{MgO}$.

O diâmetro mediano volumétrico - DMV- foi determinado por um programa computacional de um modelo matemático desenvolvido na Embrapa-CNPMA.

Para avaliar o grau de concordância entre os resultados obtidos com os dois métodos utilizados, foram ajustadas as seguintes regressões lineares: método RDAG com papel sensível versus traçador, método RDAG com $\mathrm{MgO}$ versus traçador. Essa avaliação baseou-se na magnitude do intercepto, no coeficiente de determinação e na análise de resíduos da regressão.

\section{RESULTADOS E DISCUSSÃO}

Teoricamente, se gotas de espectro uniforme fossem coletadas pela técnica da superfície de matriz graxa descrita por Matthews (1982), a aplicação do método RDAG deveria apresentar um resultado absoluto. Nesse caso, bastaria medir com precisão uma esfera de gota e multiplicar o seu volume pela densidade de gotas que atingisse o alvo. Entretanto, a precisão do método tende a diminuir quando gotas de espectro variado são coletadas em outros tipos de superfícies de amostragem, ou quando se realizar manualmente a medição, classificação e contagem das impressões das gotas

No método testado, o uso da gratícula que apresenta 14 subdivisões em progressão de $\sqrt{2}$ permite que as gotas de uma amostra sejam distribuídas em classes de tamanho, melhorando o rendimento da análise em termos de tempo. Entretanto, os cálculos são feitos com base no volume médio das gotas de cada classe, e isso pode acrescentar um erro no resultado final.

De maneira geral, os principais fatores que podem interferir na precisão são: erro humano na medição das impressões causadas pelas gotas, tanto pela dificuldade de julgamento na classificação, como na definição dos contornos das impressões; correção com fatores de espalhamento para obtenção de tamanho original; classificação em poucas faixas de tamanho; e características do alvo quanto à captura de gotas. Além desses problemas, o processo de classificação manual de gotas é extremamente cansativo. 
O ajuste dos dados utilizados para determinação de fatores de espalhamento pelo programa SAS forneceu o modelo matemático abaixo, com os seguintes parâmetros de ajuste:

$\mathrm{fe}(\mathrm{DL})=0,74057+0,0001010399 . \mathrm{DL}+0,2024884 \cdot \ln (\mathrm{DL})$, onde:

fe é o fator de espalhamento;

DL é o diâmetro-limite de cada classe de tamanho.

Os parâmetros de confiança desse modelo foram: $\mathrm{r}^{2}=0,999151$;

$\mathrm{CV}=0,320689$;

$\mathrm{Pr}>\mathrm{F}=0,001$;

$\mathrm{F}=70573,39$.

O tempo médio gasto para classificar as gotas de cada amostra ficou em torno de 30 minutos. A esse tempo devem ser somados mais 10 minutos gastos também com a determinação da densidade das gotas em cada amostra. Esses tempos foram altos em virtude do cuidado que se procurou adotar para a medição de todas as gotas, de cada campo visual selecionado no microscópio. Johnstone \& Huntington (1970), em teste de comparação de métodos de medição de gotas obtiveram, com a gratícula de Porton, tempos médios de classificação em 10 classes de tamanhos de gotas, de 5,05 minutos para um operador A e 9,58 minutos para um operador B em 6 análises. Entretanto, o coeficiente de variação médio para as 10 classes do operador A foi de $21,36 \%$, e para o operador B, 12,92\%. Segundo os autores, é necessário estabelecer um balanço entre a precisão que se deseja e o tempo, quando se necessita efetuar muitas análises; e enfatizam, que os erros cometidos na própria amostragem podem limitar a precisão de alguns dados.

Os métodos de análise química também estão sujeitos a uma série de erros, principalmente na preparação dos padrões de calibração dos aparelhos e na extração do produto do substrato. Comumente, os resultados finais das análises são corrigidos por fatores de recuperação, que, por sua vez, variam com a concentração medida.

Na Tabela 2 são apresentados os resultados da determinação dos depósitos, pelo método RDAG, para os alvos de papel sensível a água e lâminas de microscópio recobertas com $\mathrm{MgO}$, e pelo método de análise química do traçador. Pode ser observado na Fig. 1 que o volume que atingiu os quatro primeiros pontos amostrais foi menor que no restante das amostras. Isso ocorreu, provavelmente, por causa da diferença de velocidade de pulverização entre o início e o fim da aplicação.

A regressão ajustada entre o método RDAG com papel sensível (PS) e a análise do traçador (TR), resultou a seguinte equação: $\mathrm{PS}=0,9998 \mathrm{TR}$, erropadrão de 0,0158 , e coeficiente de determinação $\mathrm{r}^{2}=0,9068$. A regressão ajustada entre o método RDAG com MgO e a análise do traçador (TR), resultou na seguinte equação: $\mathrm{MgO}=0,9676$.TR, erro-padrão de 0,0149, e coeficiente de determinação $r^{2}=0,8502$. Houve boa concordância entre o método de recuperação por análise de gotas e a análise de resíduos. Entretanto, pode ser notado, pela Fig. 2, que a recuperação pela análise de gotas em amostras com $\mathrm{MgO}$ subestima os valores reais.

Um fato muito importante que deve ser levado em consideração no método RDAG, é que as gotas pequenas, apesar de não acrescentarem valores expressivos aos volumes obtidos, têm uma importância fundamental no cálculo do volume médio da gota da amostra. Existe uma relação inversa entre o número de gotas pequenas e o volume médio das gotas da amostra. Como a impressão provocada pelas gotas pequenas é pouco nítida, ou quase imperceptível no papel sensível a água, é necessário atenção muito grande para medi-las. Nesse caso, o tipo de iluminação proporcionada pelo microscópio tem importância fundamental para a análise.

A iluminação incidente foi a mais adequada para o papel sensível a água, pois aumentou a nitidez e o contraste das cores, facilitando a observação das pequenas manchas. No caso das lâminas recobertas com $\mathrm{MgO}$, uma comparação prévia entre dois tipos de iluminação demonstrou que o julgamento do tamanho das crateras produzidas pelas gotas é mais difícil sob iluminação transmitida (de baixo para cima). Sob esse tipo de iluminação, as crateras das gotas pequenas produzem um halo luminoso, proporcionando uma aparência de diâmetro maior. Por outro lado, gotas muito pequenas não conseguem penetrar totalmente na camada do $\mathrm{MgO}$, o que dificulta a sua visualização. $\mathrm{O}$ sistema de iluminação incidente (de cima para baixo), entretanto, não só possibilitou melhor nitidez para a visualização das crateras, mas 
TABELA 2. Densidade de deposição, diâmetro de gotas e volume de calda encontradas em amostras de papel sensível a água e lâmina de microscópio recoberta com $\mathrm{MgO}$ e papel-filtro.

\begin{tabular}{|c|c|c|c|c|c|c|c|}
\hline \multirow[t]{2}{*}{ Amostras } & \multicolumn{2}{|c|}{ Densidade de gotas ${ }^{1}$} & \multicolumn{2}{|c|}{$\mathrm{DMV}^{2}$} & \multicolumn{3}{|c|}{ Volume de calda $^{3}$} \\
\hline & $\mathrm{PS}^{4}$ & $\mathrm{MgO}^{5}$ & PS & $\mathrm{MgO}$ & PS & $\mathrm{MgO}$ & $\mathrm{TR}^{6}$ \\
\hline 1 & 80 & 98 & 185 & 187 & 0,1514 & 0,1538 & 0,1532 \\
\hline 2 & 65 & 63 & 193 & 238 & 0,1424 & 0,1508 & 0,1446 \\
\hline 3 & 74 & 91 & 202 & 220 & 0,2349 & 0,2390 & 0,2058 \\
\hline 4 & 94 & 110 & 206 & 222 & 0,2330 & 0,2360 & 0,2631 \\
\hline 5 & 112 & 112 & 201 & 180 & 0,2575 & 0,2430 & 0,2611 \\
\hline 6 & 98 & 142 & 212 & 183 & 0,2740 & 0,2418 & 0,2635 \\
\hline 7 & 97 & 108 & 211 & 183 & 0,2685 & 0,2561 & 0,2554 \\
\hline 8 & 160 & 134 & 176 & 174 & 0,2684 & 0,2643 & 0,2714 \\
\hline 9 & 200 & 214 & 154 & 186 & 0,2723 & 0,2621 & 0,2771 \\
\hline 10 & 143 & 183 & 169 & 187 & 0,2716 & 0,2640 & 0,2758 \\
\hline
\end{tabular}

1 Número de gotas por $\mathrm{cm}^{2}$.

2 Diâmetro mediano volumétrico $(\mu \mathrm{m})$

3 Volume $=\mu \mathrm{L} / \mathrm{cm}^{2}$

4 Papel sensível a água.

${ }^{5}$ Lâmina de microscópio recoberta com $\mathrm{MgO}$

${ }^{6}$ Volume estimado por análise química do traçador.

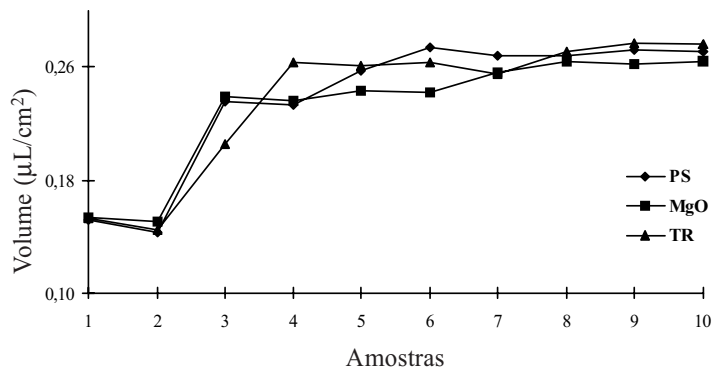

FIG. 1. Volume de calda estimada pelo método de recuperação de deposição por análise de gotas em papel sensível (PS) a água e lâminas recobertas com $\mathrm{MgO}$, e por análise química do traçador (TR).

também permitiu a observação de grande quantidade de leves depressões, com grandes diâmetros, provavelmente de gotas que ricochetearam sobre a camada do óxido. Esse fenômeno já tem sido descrito na literatura (Matthews, 1982) e pode ser considerado como uma falha do método $\mathrm{MgO}$, mas, por outro lado, não se sabe se isso também ocorre com o papel sensível a água, ou com tipos de alvos artificiais. Além das características da superfície do alvo, esse

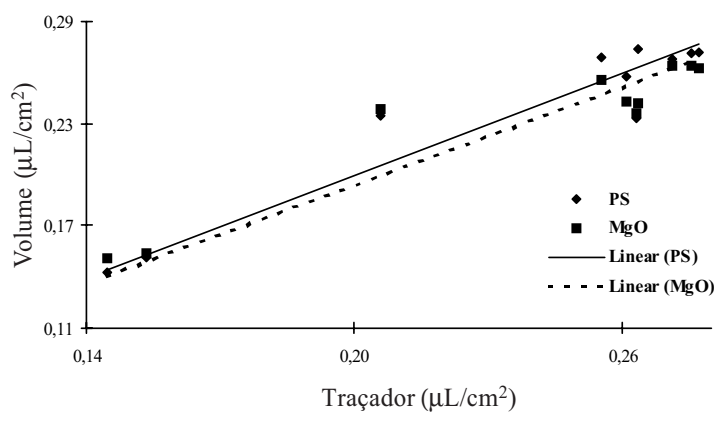

FIG. 2. Curvas obtidas a partir da análise de regressão dos valores dos volumes estimados pelo método de recuperação de deposição por análise de gotas para $\mathrm{MgO}$ e papel sensível (PS) a água com a análise química do traçador.

fenômeno também é dependente da tensão superficial do líquido, do tamanho e da força do impacto das gotas.

Saunders et al. (1976) fizeram críticas e apresentaram uma série de problemas encontrados durante as amostragens de gotas, em um estudo que realizaram com bicos para redução de deriva em pulverização aérea, com diferentes alvos artificiais. Dependendo do ângulo de incidência com que as gotas se deposi- 
tam, as impressões causadas nos alvos artificiais podem assumir formas irregulares, difíceis de serem medidas.

O ensaio realizado para testar o método RDAG foi realizado em laboratório na ausência de vento, e os problemas apontados por Saunders et al. (1976) não foram observados. Entretanto, é recomendável fazer um planejamento estatístico com base em resultados de pré-ensaios, para se estabelecer o número de amostras necessárias para obtenção de um resultado confiável, mesmo com o descarte de uma determinada porcentagem de amostras não adequadas à análise.

Os fabricantes do papel sensível a água alertam que esse substrato apresenta limitações de captura de gotas com diâmetros abaixo de $30 \mu \mathrm{m}$ de diâmetro, sob determinadas condições de umidade relativa. Entretanto, essas gotas apresentam dificuldade de deposição em qualquer tipo de alvo com superfície plana, lisa e grande. Assim, se nos experimentos para estudo de deposição de gotas forem utilizados alvos artificiais laminares e lisos, a precisão do método analítico empregado não corrigirá o erro da deficiência de captura dessas gotas.

\section{CONCLUSÕES}

1. É possível estimar a quantidade de agrotóxico que se deposita em uma determinada área através de análise de gotas, com uso de microscópio.

2. O alvo de amostragem, o fator de espalhamento, o número de classes em que as gotas são classificadas e a habilidade do técnico que efetua a análise, determinam a precisão dos resultados.

\section{REFERÊNCIAS}

CIBA-GEIGY. Water-sensitive paper for monitoring spray distribution. 3.ed. Basel: Ciba-Geigy Application Service, [19--]. 16p. (Bulletin 332-W).

CORRÊA, H.G.; MESSIAS, C.M.; CARVALHO, J.B.H.; BATAGLIA, O.C. Análise das deposições da pul- verização aérea simulando a aplicação de Metarrhizium anisopliae (Metsch) na cultura da canade-açúcar. Bragantia, Campinas, v.51, n.1, p.95-109, 1992.

DERKSEN, R.C.; JIANG, C. Automated detection of fluorescent spray deposits with a computer vision system. Transactions of the ASAE, St. Joseph, v.38, n.6, p.1647-1653, 1995.

FRANZ, E. Spray coverage analysis using a hand-held scanner. Transactions of the ASAE, St. Joseph, v.36, n.5, p.1271-1278, 1993

JIANG, G.; DERKSEN, R.C. Morphological image processing for spray deposit analysis. Transactions of the ASAE, St. Joseph, v.38, n.5, p.1581-1591, 1995.

JOHNSTONE, D.R.; HUNTINGTON, K.A. A comparison of visual microscopic methods of spray droplet size measurement using eyepieces employing the image shearing principle and the globe and circle eyepiece graticule. Journal of Agricultural Engineering Research, London, v.15, n.1, p.1-10, 1970.

HIGGINS, A. Spread factor for technical malation spray. Journal of Economic Entomology, College Park, v.60, n.1, p.280-281, 1967.

MAKSYMIUK, B.; MOORE, A.D. Spread factor variation for oil-base, aerial sprays. Journal of Economic Entomology, College Park, v.55, n.5, p.695-699, 1962.

MATTHEWS, G.A. Pesticide application methods. New York: Longman, 1982. 336p.

MAY, K.R. The measurement of airborne droplets by magnesium oxide method. Journal of Scientific Instruments, College Park, v.27, p.128-130, 1950.

SAUNDERS, W.J.; TATE, R.J.; WARE, G.W. Analysis of aerial sprays from conventional an drift reduction nozzles. St. Joseph: American Society of Agricultural Engineers, 1976. 13p. (ASAE Paper, 76-1062)

TURNER, C.J.; HUNTINGTON, A.J. The use of a water sensitive dye for the detection and assessment of smal spray droplets. Journal of Agricultural Engineering Research, London, v.15, n.4, p.385387, 1970. 